\title{
Estimation of Muscle Mass in the Integrated Assessment of Patients on Hemodialysis
}

\author{
Alice Sabatino ${ }^{1}$, Natascha J. H. Broers ${ }^{2,3}$, Frank M. van der Sande ${ }^{2,3}$, \\ Marc H. Hemmelder ${ }^{2,3}$, Enrico Fiaccadori ${ }^{1}$ and Jeroen P. Kooman ${ }^{2,3 *}$ \\ ${ }^{1}$ Nephrology Unit, Department of Medicine and Surgery, Parma University Hospital, University of Parma, Parma, Italy, \\ ${ }^{2}$ Division on Nephrology, Department of Internal Medicine, Maastricht University Medical Centre, Maastricht, Netherlands, \\ ${ }^{3}$ NUTRIM School of Nutrition and Translational Research in Metabolism, CARIM School for Cardiovascular Diseases, \\ Maastricht University, Maastricht, Netherlands
}

OPEN ACCESS

Edited by:

Michele Barone,

University of Bari Aldo Moro, Italy

Reviewed by:

Leigh C. Ward,

The University of

Queensland, Australia

Calvin Lloyd Cole,

University of Rochester, United States

*Correspondence:

Jeroen P. Kooman

Jeroen.kooman@mumc.n

Specialty section:

This article was submitted to

Clinical Nutrition

a section of the journal

Frontiers in Nutrition

Received: 19 April 2021

Accepted: 16 July 2021

Published: 16 August 2021

Citation:

Sabatino A, Broers NJH, van der

Sande FM, Hemmelder MH,

Fiaccadori E and Kooman JP (2021)

Estimation of Muscle Mass in the

Integrated Assessment of Patients on Hemodialysis. Front. Nutr. 8:697523.

doi: 10.3389/fnut.2021.697523
Assessment of muscle mass (MM) or its proxies, lean tissue mass (LTM) or fat-free mass (FFM), is an integral part of the diagnosis of protein-energy wasting (PEW) and sarcopenia in patients on hemodialysis (HD). Both sarcopenia and PEW are related to a loss of functionality and also increased morbidity and mortality in this patient population. However, loss of MM is a part of a wider spectrum, including inflammation and fluid overload. As both sarcopenia and PEW are amendable to treatment, estimation of MM regularly is therefore of major clinical relevance. Whereas, computer-assisted tomography (CT) or dual-energy X-ray absorptiometry (DXA) is considered a reference method, it is unsuitable as a method for routine clinical monitoring. In this review, different bedside methods to estimate MM or its proxies in patients on HD will be discussed, with emphasis on biochemical methods, simplified creatinine index (SCI), bioimpedance spectroscopy (BIS), and muscle ultrasound (US). Body composition parameters of all methods are related to the outcome and appear relevant in clinical practice. The US is the only parameter by which muscle dimensions are measured. BIS and SCI are also dependent on either theoretical assumptions or the use of population-specific regression equations. Potential caveats of the methods are that $\mathrm{SCl}$ can be influenced by residual renal function, BIS can be influenced by fluid overload, although the latter may be circumvented by the use of a three-compartment model, and that muscle US reflects regional and not whole body MM. In conclusion, both SCI and BIS as well as muscle US are all valuable methods that can be applied for bedside nutritional assessment in patients on HD and appear suitable for routine follow-up. The choice for either method depends on local preferences. However, estimation of MM or its proxies should always be part of a multidimensional assessment of the patient followed by a personalized treatment strategy.

Keywords: ultrasound, bioimpedance, sarcopenia, hemodialysis, creatinine index

\section{INTRODUCTION}

Assessment of nutritional state is of high relevance in patients on hemodialysis (HD). This given the relation between protein-energy wasting (PEW), a condition characterized by reduced body stores of protein and energy fuels characteristic of patients with chronic kidney disease (CKD) and end-stage kidney disease (ESKD), and mortality (1), and since abnormalities in the nutritional state may be amenable to therapeutic intervention (2). Muscle wasting in patients on HD can be due to 
multiple factors including insufficient dietary intake and a loss of nutrients through the dialysate, or an increased muscle breakdown due to inflammation or metabolic acidosis (3). Measurement of muscle mass (MM), or its proxies lean tissue mass (LTM) or fat-free mass (FFM), is an integral part of the assessment of the nutritional state, as well as in the diagnosis of sarcopenia. Sarcopenia is characterized by reduced MM and strength and is frequently observed in the elderly, but can also happen earlier as a consequence of chronic conditions, such as CKD/ESKD and patients on HD (3-5). Malnutrition as well as sarcopenia are part of a spectrum including impaired functional status, low physical activity, low quality of life, and frailty (68 ), and they are the important components of the premature aging phenomenon in this patient population (9). Next to this, inflammation and fluid overload were also found to be related to a decrease in LTM and intracellular water $\operatorname{ICW}(8,10)$. Thus, loss of MM is a central part of the multimorbid spectrum of patients on $\mathrm{HD}$, and should be interpreted in view of both its consequences as well as in the context of potentially amendable underlying factors. The aim of this short review is to give a concise overview of instrumental methods that can be used on a daily clinical basis in patients on $\mathrm{HD}$, and their use in the context of a multidimensional assessment in these patients will be discussed.

\section{CLINICAL SYNDROMES ASSOCIATED WITH A LOSS OF MM}

Loss of MM or its proxies is included in various syndromes related to the nutritional and functional status of the patient on HD, as summarized in Table 1. Except frailty, in which only a reduction in muscle strength is a parameter, a reduction of MM or FFM is included in the diagnostic criteria of other syndromes, such as PEW, cachexia, and sarcopenia. These partly, but not entirely, overlapping syndromes $(3,11)$ are part of a wide spectrum of nutritional and functional abnormalities in patients on $\mathrm{HD}$, although an important common denominator appears to be tissue loss (11). Importantly, one of the criteria for the definition of PEW, also referred to as kidney cachexia, is an increase in inflammatory parameters $(4,12)$. In contrast, inflammation is not included in the diagnostic criteria of sarcopenia (13). This division is relevant, as the pathophysiology and also possibly the clinical approach to a patient on HD with a pure "sarcopenic" phenotype may differ from that of a patient with a "cachectic" phenotype (14). Furthermore, patients can have both an increase in fat mass and a decline in MM (15), an entity also known as sarcopenic obesity, which is prevalent in patients on $\mathrm{HD}$, although its relation with the outcome is yet uncertain $(16,17)$. The development of sarcopenic obesity is not captured by the estimation of changes in body weight or body mass index (BMI) (18).

The assessment of body composition is complicated by the fact that various parameters are used to express (loss) of MM or LBM. For instance, LBM, FFM, and MM are not equivalent, although they are often used as interchangeable surrogates. FFM, as the name suggests, is the total body mass except for the body fat, and it includes LBM and bone mineral tissue. The LBM, in turn, is composed of the total body water, appendicular skeletal muscle mass (ASMM), and the fat-free mass of organs. Since different techniques measure different compartments, the identification of the body compartment of interest, along with the availability of the method, must precede the choice of the method of assessment. As an example, some available techniques, such as bioelectrical impedance analysis (BIA), assess body composition by dividing the body into two compartments (2C), the FFM, which conceptually includes all non-fat tissue and the fat mass (FM) (19). While other methods, such as dualenergy X-ray absorptiometry (DXA) and one application of bioelectrical impedance spectroscopy (BIS), divides the body into three compartments (3C). DXA assesses LBM, which includes total body protein and total body water (TBW), but excludes bone and fat mass $(20,21)$ (Figure 1). On the other hand, 3C-BIS assess a "normohydrated" LTM, which reflects a compartment that is separate from adipose tissue mass (ATM; fat mass and adipose water) and a virtual "overhydration" compartment, but that includes bone mineral tissue (8) (Figure 1), as will be discussed later in more detail.

These specific examples show that although all entities reflect comparable physiological dimensions, they are not interchangeable. FFM assessed by a $2-\mathrm{C}$ model can be different from LTM assessed by a 3-C model (22). Thus, parameters obtained by a specific method cannot be used interchangeably with comparable parameters assessed by different methodologies. In addition, ideally, reference values should also be developed for specific techniques, devices, and populations. As this may not always be feasible, it is important to have these caveats in mind when assessing literature on body composition in patients on kidney replacement therapy (KRT).

\section{BEDSIDE TECHNOLOGY FOR THE ASSESSMENT OF MM}

For the assessment of $\mathrm{MM}$ or its proxies, various options are available. Computer-assisted tomography (CT) or MRI are considered gold standard methods but are impractical to be used on a routine basis $(13,23)$. DXA is generally considered a reference method to estimate LBM as well as ASMM in guidelines (13) but may be difficult to perform frequently in clinical practice. Furthermore, because DXA assumes a hydration ratio with LBM of 0.73 (24), results can be influenced by severe fluid overload (25). Still, DXA, when routinely available, provides important information on changes in body composition on HD and might also serve as a calibration for bedside methods.

Various methods are available to assess MM or LBM in patients which can be used on a routine basis in patients on KRT. These can be conceptually divided into methods that indirectly estimate body composition (such as bioimpedance or anthropometry), biochemical methods (based on creatinine kinetics), and methods that measure muscle dimensions at an anatomical level [MRI, CT, or ultrasound (US)]. Anthropometry is a time-honored method that is also included in the original diagnostic criteria for PEW (4). When performed by a skilled 
TABLE 1 | Categories of assessment for the diagnosis of different syndromes related to the nutritional and functional status of patients.

\begin{tabular}{|c|c|c|c|c|c|c|}
\hline Criteria & Malnutrition ESPEN & Malnutrition GLIM & PEW & Cachexia & Sarcopenia & Frailty \\
\hline Weight loss/BMl & + & + & + & + & & + \\
\hline Muscle mass/FFM/LTM & + & + & + & + & + & \\
\hline Muscle strength & & & & & + & + \\
\hline Functional performance & & & & & + & + \\
\hline Fatigue/exhaustion & & & & & & + \\
\hline Biochemical markers & & & + & + & & \\
\hline Etiology & & + & & & & \\
\hline
\end{tabular}

BMI, body mass index; FFM, fat free mass; LTM, lean tissue mass; PEW, protein energy wasting.

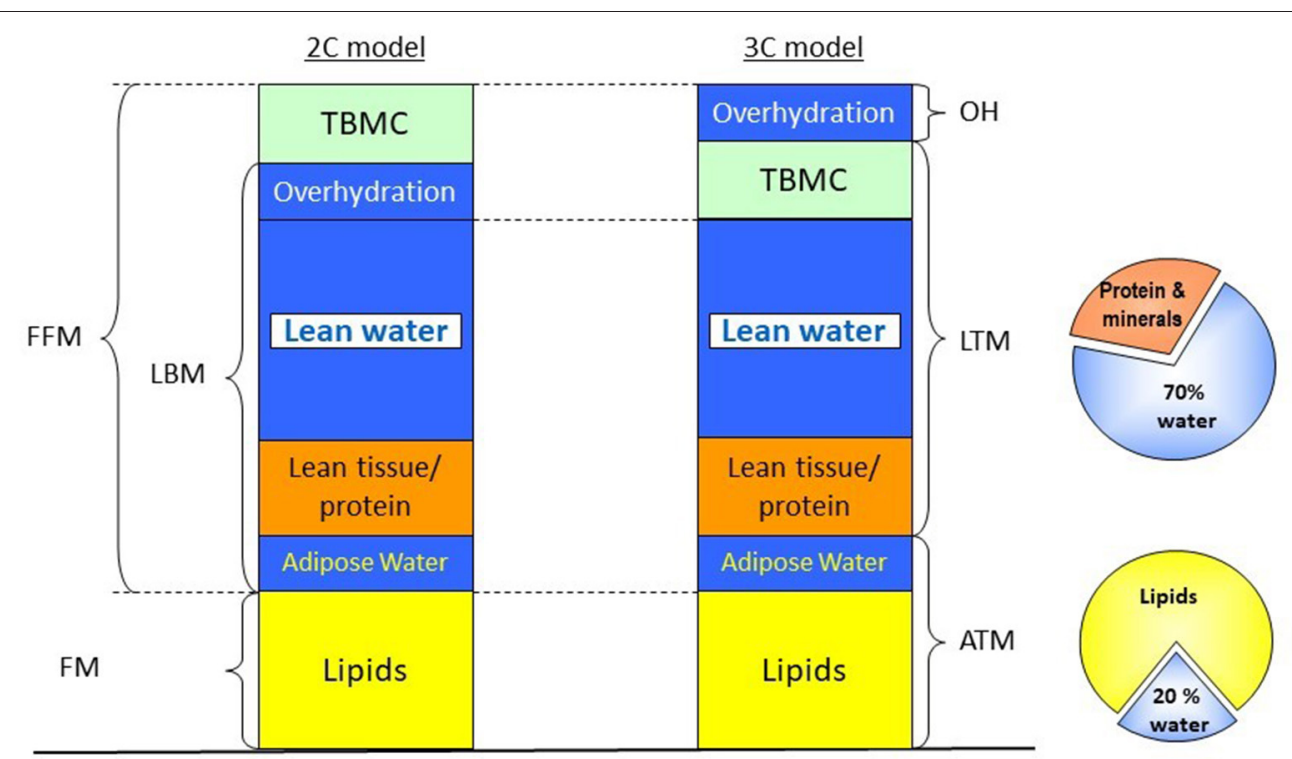

FIGURE 1 | Different body compartment models based on BIA, BIS, and DXA. In the BIA approach, a two-compartment model is applied, dividing the body in FFM, which includes bone mineral tissue, total body water, skeletal muscle and visceral proteins, and FM. Both DXA and three-compartment BIS apply a three-compartment approach; however, in the case of DXA, bone mineral density is removed from the FFM, and LBM is measured instead, while in the case of BIS, a virtual "overhydration" compartment is calculated as the difference between measured and expected ECW, providing information on ATM and a normohydrate LTM, which includes bone mineral tissue. ATM, adipose tissue mass; BIA, bioelectrical impedance analysis; BIS, bioelectrical impedance spectroscopy; DXA, dual-energy X-ray absorptiometry; FFM, fat-free mass; FM, fat mass; LBM, lean body mass; LTM, lean tissue mass; OH, overhydration; TBMC, total body mineral content.

Modified from Broers et al. (22) with permission.

investigator it was able to predict a reduction in MM with an accuracy comparable with instrumental methods (16). However, the emphasis of the present article is on biochemical and technological tools in the assessment of body composition.

\section{Bioelectrical Impedance}

Although the theoretical backgrounds of BIA are complex and discussed in excellent reviews $(26,27)$, in general, this method measures the opposition (impedance) of the body or a body segment to an alternating current. The impedance $(Z)$ is a composite of the resistance to this flow, which is related to TBW, and reactance $(\mathrm{Xc})$ is related to the capacitance of the cellular membrane. With the single-frequency (SF) approach, FFM and ASMM are estimated using population-derived regression equations, including $\mathrm{Z}$ or $\mathrm{R}$, measured at $50 \mathrm{kHz}$ as the resistance index $\mathrm{H}^{2} / \mathrm{R} 50$ along with anthropometric parameters, sex, and age (27). With SF-BIA, also ASMM can be estimated using regression equations $(27,28)$.

The multifrequency (MF) approach delivers different frequencies that vary from 5 to $1,000 \mathrm{kHz}$, and depending on the method it can use only several frequencies or, in the case of BIS, broadband of frequencies within this range. The lower the frequency, the more the difficulty with which it has to pass across the cell, passing only at the extracellular water (ECW) at frequencies $<$ than $1 \mathrm{kHz}$ and not through the ICW, with higher frequencies it passes through both, with TBW being measured at frequencies $>5,000 \mathrm{kHz}$. For technical reasons, measurements at very low and very high frequencies are not possible; however, with BIS, the resistance at zero (Ro) and infinity $(\mathrm{R} \infty)$ are extrapolated by applying a Cole-Cole plot to predict ECW and TBW (27). In classic 2C-models of SF-BIA and MF-BIA, FFM is subsequently calculated from TBW, assuming fractional 
hydration of 0.73 (29). A drawback of this method, which divides the body into two compartments, is that the excess of ECW due to fluid overload is added to the TBW which can subsequently result in an overestimation of FFM (25) (Figure 1). In the case of BIS, Moissl et al. developed a model for the assessment of ECW, ICW, and TBW using Hanai mixture theory adjusted for BMI, which showed good agreement with dilution methods (30). Chamney et al. further developed the so-called 3C-model, which assumes fixed hydration of LTM and ATM, and divides the body in normohydrate LTM and ATM, and a virtual "overhydration" (OH) compartment (31).

With regard to the estimation of body composition, a definite superiority of either approach has not yet been proven. Donadio et al. found a slightly lower prediction error for FFM with SFBIA as compared with an MF-BIA using a two-compartment approach, with a highly significant correlation of both methods with DXA (32). Another study observed a stronger relation between FFM estimated by MF-BIA and creatinine kinetics as compared with SF-BIA (33). Raimann et al. found a slightly improved estimation of ICW with the SF method, and conversely, improved estimation of ECW with BIS. With regard to the detection of changes in ECW, MF-BIA was found to have a higher precision $(34,35)$. On the other hand, ASMM predicted by the Sergi equation using SF-BIA showed high accuracy in predicting sarcopenia with DXA as the reference method (16); however, this equation with the form: ASMM $(\mathrm{kg})=-3.964+\left(0.227^{*}\right.$ [height $\left.\left.(\mathrm{cm})]^{2} / \mathrm{R}\right]\right)+\left(0.095^{*}\right.$ weight $)+\left(1.384^{*}\right.$ sex $)+\left(0.064^{*} \mathrm{Xc}\right)$, was primarily validated in an elderly Caucasian population (36).

With the 3C BIS method, estimation of body composition is based on a theoretical approach without the use of populationspecific regression equations. Lean tissue index (LTI), which corresponds to LTM, divided by height ${ }^{2}$, below the 10th percentile of a healthy age-matched reference population was independently related to outcome in most, but not all studies (8, $15,37,38)$. Still, in a meta-analysis including over 15,000 patients, a low LTI was associated with increased mortality [Hazard ratio 1.53 (95\% CI: 1.41-1.64)] (39). Especially, the combination of a low LTI and fat tissue index (FTI, the height ${ }^{2}$-normalized ATM) appears to be associated with increased mortality risk (15).

However, in several studies, despite reasonable agreement at a population level, relatively wide limits of agreement were observed between body compartments assessed by $3 \mathrm{C}$-BIS and reference techniques, such as DXA $(40,41)$. To some degree, these differences may be explained by the fact that even the reference method is not free of errors. Indeed, the excess ECW with overhydration is added to the LBM compartment with DXA, but not with the 3C-BIS approach (8). Also, it should be taken into account that ATM assessed by 3C-BIS includes intra adipose water, unlike FM measured by DXA. Using ASMM measured by DXA as the reference method, LTM measured by wholebody BIS was able to predict sarcopenia with acceptable accuracy (mean AUC 0.79 for females and 0.77 for males) (16); however, it should be noted that in the current EWGSOP2 guidelines, cutoff values based on the 3C-BIS model were not yet included in the definition, while the Sergi equation based on SF BIA was advocated for standardization (13). However, in the case of tissue edema, these estimations may be less reliable in patients on HD.
To standardize measurements and avoid this kind of problem, the recent KDOQI guidelines on nutrition in CKD recommends that BIA/BIS should be performed at least $30 \mathrm{~min}$ after the HD session to allow for the distribution of body fluids (42). Still, in the case of 3C-BIS, measurements of ATM and normohydrated LTM were slightly $(0.77$ and $0.40 \mathrm{~kg}$, respectively) affected by the timing of measurements (43), whereas predialytic fluid status was more consistently related to the outcome as compared with postdialytic measurements (44).

An alternative approach is the construction of a vector plotting $\mathrm{R}$ and $\mathrm{Xc}$ at $50 \mathrm{kHz}$ within tolerance ellipses of a healthy population. The advantage of this method is that results are displayed without the need for underlying theoretical assumptions or population-based equations (28). A potential disadvantage is that the direct translation of the findings into constructs such as sarcopenia, fluid overload, and PEW may be more difficult as compared with a numerical approach.

To summarize, whereas various BIA approaches can be used to assess body composition in dialysis patients, it must be kept in mind that estimations are dependent on population-specific regression equations or theoretical assumptions regarding the conversion from bioelectrical signals to estimations of body water compartments. Measurements obtained with a specific device or method can therefore not be used interchangeably (45), even with regard to raw parameters such as Z, Xc, and R (46). It is important to acknowledge that as long as a device is correctly calibrated, the magnitude of the differences are small and generally within the precision of the specifications of the manufacturer. Whereas, a definite superiority of a specific BIA methodology for estimations of body composition has not been proven, in our view, the 3$\mathrm{C}$ model holds the advantage that it also provides a separate estimation of fluid status in a single measurement, whereas it has shown high-construct validity in predicting outcome in large datasets.

\section{Biochemical Methods: The Creatinine Index}

Serum creatinine is a breakdown product of creatine phosphate in muscle tissue that was found to be strongly related to LBM assessed by DXA in patients on HD (47). Serum creatinine is inversely related to mortality in patients on renal replacement therapy (48); however, serum creatinine in patients on HD is also dependent on dialysis adequacy. Therefore, the concept of creatinine index (CI) was developed (49). CI was found to be an independent predictor of outcome in patients with HD (50). However, as creatinine kinetics may be complicated to use in routine clinical practice, a simplified form [simplified creatinine index (SCI)] was developed, which was also found to be related to the outcome (51). SCI $(\mathrm{mg} / \mathrm{kg} / \mathrm{d})$ is calculated according to the formula $16.21+1.12^{*}$ [ 1 if male; 0 if female] $-0.06^{*}$ age (years) $-0.08^{*} \mathrm{spKt} / \mathrm{V}$ urea $+0.009^{*}$ predialytic serum creatinine $(\mu \mathrm{mol} / \mathrm{l})$. Also, LTI derived from SCI was strongly related to LTI assessed by BIS, although the mean BIS-derived value was $4.7 \mathrm{~kg}$ lower than the SCI-estimated value (51). SCI is easy to apply in clinical practice as only routinely gathered data that are already present in electronic health records (EHR) are needed, 
with the advantage that longitudinal trends can be tracked easily. Indeed, SCI declined 6 months before death, potentially serving as an early warning sign (51). However, a potential pitfall in the follow-up of the SCI is changes in residual renal function, which may independently affect serum creatinine values apart from muscle mass, as well as changes in dietary intake (42). Serum creatinine is also a parameter included in the nutritional component score (NCS), an aggregate score that also consists of routinely captured parameters interdialytic weight gain, serum phosphate, serum albumin, and normalized protein catabolic rate (nPCR). The use of this score, which was shown to decline 1-2 months before hospitalization and also up to 6 months before death $(52,53)$, allows for the interpretation of changes in parameters like interdialytic weight gain (IDWG) and serum phosphate, which have a bidirectional relationship with the outcome (54). Whereas, a decrease in these parameters is usually regarded as a positive sign given their detrimental effect on the cardiovascular status of the patient, a sharp decline in IDWG and serum phosphate can also be a sign of impending malnutrition and adverse outcomes when accompanied by a decrease in the other nutritional parameters (52).

To Summarize, following trends in biochemical indices derived from EHR can aid in the early detection of changes in nutritional state and $\mathrm{MM}$, whereas changes in serum creatinine or SCI cannot replace validated questionnaires to establish the risk for sarcopenia (55), they can aid in case finding given the fact that they can be performed on a frequent and routine basis with the potential for automated processing of the findings.

\section{Muscle Ultrasound}

Recently, regional muscle US has been applied in patients with kidney disease for the assessment and monitoring of skeletal muscle. Its major advantages, compared to other imaging techniques, are represented by lower cost, portability, lack of radiation exposure, and the possibility to be applied by non-specialized staff (56-58). In comparison to other bedside techniques, such as anthropometry, US allows realtime visualization of the target structure, allowing for the assessment of muscle size (thickness and area) and/or quality, through echogenicity, which provides information about the presence of inflammation, fibrosis, and adipose infiltration (59). Its portability is of particular interest in the CKD research setting and clinical practice since patients can be evaluated during HD session or outpatient visits.

Quadriceps muscle US has been studied extensively in patients with renal disease. In the available research, two muscles were most frequently studied, the quadriceps rectus femoris (RF) and vastus intermedius (VI), in two different points, the midpoint, and at the border of the lower third and upper two-thirds between the anterior superior iliac spine and the upper pole of the patella (Figure 2) (56, 57, 60-62). Abundant contact gel to avoid any pressure is needed to prevent muscle deformation. Regarding the accuracy and reproducibility of the method, its reliability has been tested in critically ill patients with acute kidney injury (AKI), showing excellent intraclass correlation coefficients (ICC) for inter- and intra-operator comparisons, as well as for measurements performed before and after HD (56).

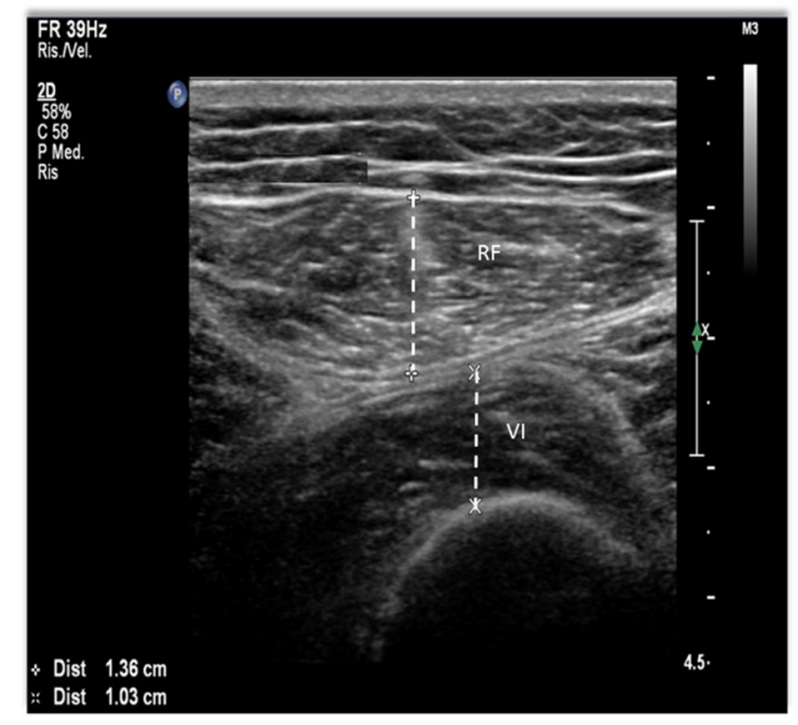

FIGURE 2 | Quadriceps muscle ultrasound (US) methodology. The points of interest correspond to the midpoint and the lower third between the anterior superior iliac spine (ASIS) and the upper pole of the patella. Using a B-mode ultrasound with a linear transducer, we obtain the image on the right. Rectus femoris (RF) and vastus intermedius (VI) thickness, a measure on the inner edge of the muscle.

In the same clinical setting, US assessment of quadriceps muscle has also been validated against CT (60), showing small and nonsignificant differential and proportional bias in comparison with CT (60). Also in patients with AKI, US was successfully used to monitor the quadriceps muscle in the first 5 days of stay in the intensive care unit, being able to identify early muscle loss (63). In non-acutely ill patients, US was performed in patients on HD before and after the dialysis session to assess whether the presence of fluid overload or the rapid fluid shifts caused by the treatment could influence measurements (57). No differences were found between measurements performed before and after dialysis, and the correlation between measurements was very high, ranging from 0.91 to 0.95 (57), showing that muscle US is not influenced by fluid overload. Also in the outpatient setting, the assessment of muscle cross-sectional area (CSA) was validated using CT in patients with CKD and not on HD (61). In another study, RF-CSA was assessed before and after 12 weeks of resistance exercise in patients with CKD not on $\mathrm{HD}$, showing a high correlation with MRI at baseline and follow-up, and moderate positive association observed between changes in RFCSA by US and quadriceps volume by MRI following exercise training (64).

More recently, studies investigating the role of quadriceps muscle US in identifying patients with PEW or sarcopenia have been published. Sabatino et al. (57) used the PEW cutoffs for BMI and albumin to stratify patients on chronic $\mathrm{HD}$ in two groups and found that in the multivariable analysis patients with lower BMI had lower muscle thickness, whereas no difference was found between patients with serum albumin 


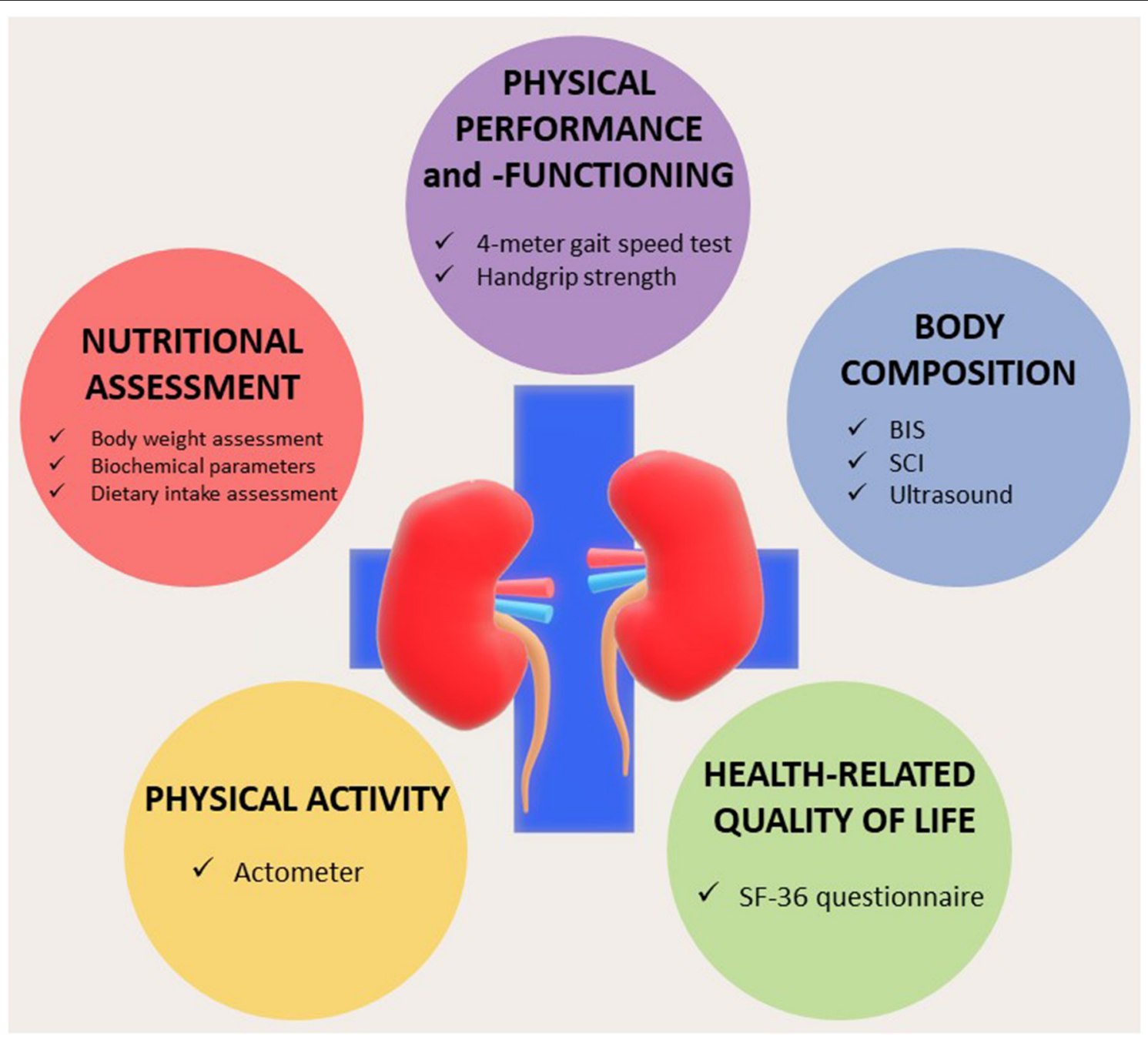

FIGURE 3 | Dashboard summarizing the integrated nutritional assessment of patients with chronic kidney disease (CKD)/end-stage kidney disease (ESKD). BIS, bioelectrical impedance spectroscopy; SCl, simplified creatinine index. Modified from Broers et al. (8) under Creative Commons.

below or above the reference value (57). A similar analysis was performed using the malnutrition inflammation score (MIS), an internationally recognized tool to assess malnutrition and predict outcomes in patients on $\mathrm{HD}$, and found that patients with worse scores ( $\geq 6$ ) had lower RF and VI thickness (57). In another study, quadriceps CSA cut-offs have been derived using receiver-operation characteristic curves based on the presence or absence of PEW in a Malaysian population (62). In that study, patients diagnosed with PEW had significantly lower RF and VI thickness and RF CSA in comparison with wellnourished patients, and the area under the curve (AUC) for RF CSA was high (men $=0.74,95 \%$ CI: $0.66-0.82$ and women $=0.82$, 95\% CI: $0.73-0.91$, both $p<0.001$ ). In addition, the correlation between US and LTI by BIS ranged from moderate to high (0.28-0.52) depending on the measurement site, with a higher correlation for RF thickness and CSA in comparison to VI.
Despite such encouraging results, more work is needed before assuming muscle US as a reference method for the diagnosis of sarcopenia. Studies defining reference values derived from healthy subjects from populations with different ethnic backgrounds should be performed to allow the early identification of patients with low MM. However, considering its validity, reliability, and sensitivity in detecting changes in skeletal muscle, its use as a tool for the monitoring of the regional muscularity of a patient could be recommended.

\section{The Role of Estimation of MM in the Integrated Functional Assessment of Patients on KRT}

Assessment of MM or its derivatives achieves its full potential in combination with other parameters. As shown in Table 1, with a relatively limited battery of measurements, various clinical 
syndromes such as PEW, sarcopenia, and frailty can be easily diagnosed. For the diagnosis of sarcopenia, it should be combined with an assessment of muscle strength, for instance, handgrip strength (HGS), and in case of a positive diagnosis, with a measure of physical function such as the $4 \mathrm{~m}$ gait speed test (13). HGS and the gait speed tests are easy to perform even in a routine clinical setting. Muscle quantity and strength, though interrelated (22), are not equivalent. Muscle strength appears to be a more powerful predictor for the outcome as compared to MM (65). Furthermore, following renal transplantation, we observed a profound increase in HGS without a significant increase in LTM (66).

Which bedside test should be used for the assessment of MM/FFM/LBM depends on local preferences and availability. In the case of 3C-BIS, information on body composition as well as the fluid overload is combined in a single measurement. Muscle ultrasound may be superior to BIS in the diagnosis of skeletal muscle depletion, but reference values need to be defined in larger populations, whereas a trained investigator is necessary. A possibility is to use SCI or BIS for case finding, followed by the US for a more precise estimate of MM depletion.

A diagnosis of PEW or sarcopenia should be combined with an estimate of potentially modifiable factors such as dietary intake and physical activity, for example, by performing actimetry regularly. Also, impaired physical activity, nutritional status, and physical performance should be interpreted given its relation with a low-health-related QoL $(7,67)$, which is especially important as these factors are often amenable to therapeutic intervention. Lastly, complications that are frequently associated with loss of MM, such as inflammation and fluid overload, should be assessed. A proposal for an integrated assessment, preferably summarized in an easily interpretable dashboard, is illustrated in Figure 3. Such a dashboard could be the basis for a personalized

\section{REFERENCES}

1. Moreau-Gaudry X, Jean G, Genet L, Lataillade D, Legrand E, Kuentz F, et al. A simple protein-energy wasting score predicts survival in maintenance hemodialysis patients. J Ren Nutr. (2014) 24:395-400. doi: 10.1053/j.jrn.2014.06.008

2. Sabatino A, Piotti G, Cosola C, Gandolfini I, Kooman JP, Fiaccadori E. Dietary protein and nutritional supplements in conventional hemodialysis. Semin Dial. (2018) 34:583-91. doi: 10.1111/sdi.12730

3. Sabatino A, Cuppari L, Stenvinkel P, Lindholm B, Avesani CM. Sarcopenia in chronic kidney disease: what have we learned so far? J Nephrol. (2020). doi: 10.1007/s40620-020-00840-y. [Epub ahead of print].

4. Fouque D, Kalantar-Zadeh K, Kopple J, Cano N, Chauveau P, Cuppari L, et al. A proposed nomenclature and diagnostic criteria for protein-energy wasting in acute and chronic kidney disease. Kidney Int. (2008) 73:391-8. doi: 10.1038/sj.ki.5002585

5. Noce A, Marrone G, Ottaviani E, Guerriero C, Di Daniele F, Pietroboni Zaitseva A, et al. Uremic sarcopenia and its possible nutritional approach. Nutrients. (2021) 13:147. doi: 10.3390/nu130 10147

6. Kim JC, Kalantar-Zadeh K, Kopple JD. Frailty and protein-energy wasting in elderly patients with end stage kidney disease. J Am Soc Nephrol. (2013) 24:337-51. doi: 10.1681/ASN.2012010047

7. Gadaen RJR, Kooman JP, Cornelis T, van der Sande FM, Winkens BJ, Broers $\mathrm{NJH}$. The effects of chronic dialysis on physical status, quality of life, and approach. For instance, a patient with a low LTI or MM and reduced muscle strength with adequate nutritional intake and absence of inflammation (a "sarcopenic" phenotype), but with low physical activity may primarily benefit from both aerobic as well as resistance training (68). In addition, activity trackers or smartphone applications may provide the patient with feedback on his/her physical activity patterns.

On the other side of the spectrum, a patient with a "cachectic" phenotype, with inflammation, and reduced protein intake, will primarily benefit from a search into the cause of inflammation and targeted nutritional intervention. Participation in an active rehabilitation program will be much more difficult for this patient, although interventions such as neuromuscular electrical stimulation may be beneficial (69). In addition, the risk of fluid overload may be increased in this patient, which may only be resolved by an increase in dialysis time due to the altered distribution between the interstitial and intravascular compartment (70).

In conclusion, a reduction in MM is an important determinant of various clinical syndromes in patients on dialysis, which are related to increased morbidity and mortality but also potentially amenable to therapeutic intervention. Whereas, different bedside methods can be used to assess MM or its proxies in patients on dialysis, it is important to maintain a critical view of their relative advantages and potential pitfalls. Assessment of MM should be part of a multidimensional approach and a personalized treatment strategy.

\section{AUTHOR CONTRIBUTIONS}

AS and JK conceived the paper. NB, FS, MH, and EF contributed to and reviewed the paper. All authors contributed to the article and approved the submitted version. arterial stiffness: a longitudinal study in prevalent dialysis patients. Nephron. (2021) 145:44-54. doi: 10.1159/000510624

8. Broers NJH, Canaud B, Dekker MJE, van der Sande FM, Stuard S, Wabel $\mathrm{P}$, et al. Three compartment bioimpedance spectroscopy in the nutritional assessment and the outcome of patients with advanced or end stage kidney disease: what have we learned so far? Hemodial Int. (2020) 24:148-61. doi: $10.1111 /$ hdi.12812

9. Kooman JP, Kotanko P, Schols AM, Shiels PG, Stenvinkel P. Chronic kidney disease and premature ageing. Nat Rev Nephrol. (2014) 10:732-42. doi: 10.1038/nrneph.2014.185

10. Gracia-Iguacel C, Gonzalez-Parra E, Mahillo I, Ortiz A. Low intracellular water, overhydration, and mortality in hemodialysis patients. J Clin Med. (2020) 9:3616. doi: 10.3390/jcm9113616

11. Gingrich A, Volkert D, Kiesswetter E, Thomanek M, Bach S, Sieber CC, et al. Prevalence and overlap of sarcopenia, frailty, cachexia and malnutrition in older medical inpatients. BMC Geriatr. (2019) 19:120. doi: 10.1186/s12877-019-1115-1

12. McKeaveney C, Slee A, Adamson G, Davenport A, Farrington K, Fouque D, et al. Using a generic definition of cachexia in patients with kidney disease receiving haemodialysis: a longitudinal (pilot) study. Nephrol Dial Transplant. (2020). doi: 10.1093/ndt/gfaa174. [Epub ahead of print].

13. Cruz-Jentoft AJ, Bahat G, Bauer J, Boirie Y, Bruyere O, Cederholm T, et al. the extended Group for: Sarcopenia: revised European consensus on definition and diagnosis. Age Ageing. (2019) 48:16-31. doi: 10.1093/ageing/ afy 169 
14. Ali S, Garcia JM. Sarcopenia, cachexia and aging: diagnosis, mechanisms and therapeutic options - a mini-review. Gerontology. (2014) 60:294-305. doi: $10.1159 / 000356760$

15. Marcelli D, Usvyat LA, Kotanko P, Bayh I, Canaud B, Etter M, et al. Body composition and survival in dialysis patients: results from an international cohort study. Clin J Am Soc Nephrol. (2015) 10:1192-200. doi: 10.2215/CJN.08550814

16. Bellafronte NT, Sizoto GR, Vega-Piris L, Chiarello PG, Cuadrado GB. Bedside measures for diagnosis of low muscle mass, sarcopenia, obesity, and sarcopenic obesity in patients with chronic kidney disease under non-dialysisdependent, dialysis dependent and kidney transplant therapy. PLoS ONE. (2020) 15:e0242671 doi: 10.1371/journal.pone.0242671

17. Malhotra R, Deger SM, Salat H, Bian A, Stewart TG, Booker C, et al. Sarcopenic obesity definitions by body composition and mortality in the hemodialysis patients. J Ren Nutr. (2017) 27:84-90. doi: 10.1053/j.jrn.2016.09.010

18. Marcelli D, Brand K, Ponce P, Milkowski A, Marelli C, Ok E, et al. Longitudinal changes in body composition in patients after initiation of hemodialysis therapy: results from an international cohort. J Ren Nutr. (2016) 26:72-80. doi: 10.1053/j.jrn.2015.10.001

19. Franssen FM, Rutten EP, Groenen MT, Vanfleteren LE, Wouters EF, Spruit MA. New reference values for body composition by bioelectrical impedance analysis in the general population: results from the UK Biobank. J Am Med Dir Assoc. (2014) 15:448 e1-6. doi: 10.1016/j.jamda.2014. 03.012

20. Prado CM, Heymsfield SB. Lean tissue imaging: a new era for nutritional assessment and intervention. J Parenter Enteral Nutr. (2014) 38:940-53. doi: 10.1177/0148607114550189

21. Mundi MS, Patel JJ, Martindale R. Body composition technology: implications for the ICU. Nutr Clin Pract. (2019) 34:48-58. doi: 10.1002/ncp.10230

22. Broers NJ, Martens RJ, Cornelis T, Diederen NM, Wabel P, van der Sande FM, et al. Body composition in dialysis patients: a functional assessment of bioimpedance using different prediction models. J Ren Nutr. (2015) 25:121-8. doi: 10.1053/j.jrn.2014.08.007

23. Sabatino A, D'Alessandro C, Regolisti G, di Mario F, Guglielmi G, Bazzocchi A, et al. Muscle mass assessment in renal disease: the role of imaging techniques. Quant Imaging Med Surg. (2020) 10:1672-86. doi: 10.21037/qims.2020.03.05

24. Salamone LM, Fuerst T, Visser M, Kern M, Lang T, Dockrell M, et al. Measurement of fat mass using DEXA: a validation study in elderly adults. J Appl Physiol. (2000) 89:345-52. doi: 10.1152/jappl.2000.89.1.345

25. Konings CJ, Kooman JP, Schonck M, van Kreel B, Heidendal GA, Cheriex $\mathrm{EC}$, et al. Influence of fluid status on techniques used to assess body composition in peritoneal dialysis patients. Perit Dial Int. (2003) 23:184-90. doi: $10.1177 / 089686080302300215$

26. Kotanko P, Levin NW, Zhu F. Current state of bioimpedance technologies in dialysis. Nephrol Dial Transplant. (2008) 23:808-12. doi: 10.1093/ndt/gfm889

27. Khalil SF, Mohktar MS, Ibrahim F. The theory and fundamentals of bioimpedance analysis in clinical status monitoring and diagnosis of diseases. Sensors. (2014) 14:10895-928. doi: 10.3390/s140610895

28. Lukaski HC. Evolution of bioimpedance: a circuitous journey from estimation of physiological function to assessment of body composition and a return to clinical research. Eur J Clin Nutr. (2013) 67(Suppl. 1):S2-9 doi: 10.1038/ejcn.2012.149

29. Molfino A, Don BR, Kaysen GA. Comparison of bioimpedance and dualenergy $\mathrm{x}$-ray absorptiometry for measurement of fat mass in hemodialysis patients. Nephron Clin Pract. (2012) 122:127-33 doi: 10.1159/000350817

30. Moissl UM, Wabel P, Chamney PW, Bosaeus I, Levin NW, BosyWestphal A, et al. Body fluid volume determination via body composition spectroscopy in health and disease. Physiol Meas. (2006) 27:921-33. doi: 10.1088/0967-3334/27/9/012

31. Chamney PW, Wabel P, Moissl UM, Muller MJ, Bosy-Westphal A, Korth $\mathrm{O}$, et al. A whole-body model to distinguish excess fluid from the hydration of major body tissues. Am J Clin Nutr. (2007) 85:80-9. doi: 10.1093/ajcn/ 85.1 .80

32. Donadio C, Halim AB, Caprio F, Grassi G, Khedr B, Mazzantini M. Single- and multi-frequency bioelectrical impedance analyses to analyse body composition in maintenance haemodialysis patients: comparison with dual-energy x-ray absorptiometry. Physiol Meas. (2008) 29:S517-24 doi: 10.1088/0967-3334/29/6/S43

33. Donadio C, Consani C, Ardini M, Bernabini G, Caprio F, Grassi G, et al. Estimate of body water compartments and of body composition in maintenance hemodialysis patients: comparison of single and multifrequency bioimpedance analysis. J Ren Nutr. (2005) 15:332-44. doi: 10.1016/j.jrn.2005.04.001

34. Raimann JG, Abbas SR, Liu L, Zhu F, Larive B, Kotanko P, et al. Agreement of single- and multi-frequency bioimpedance measurements in hemodialysis patients: an ancillary study of the frequent hemodialysis network daily trial. Nephron Clin Pract. (2014) 128:115-26. doi: 10.1159/000366447

35. Raimann JG, Zhu F, Wang J, Thijssen S, Kuhlmann MK, Kotanko P, et al. Comparison of fluid volume estimates in chronic hemodialysis patients by bioimpedance, direct isotopic, dilution methods. Kidney Int. (2014) 85:898908. doi: 10.1038/ki.2013.358

36. Sergi G, De Rui M, Veronese N, Bolzetta F, Berton L, Carraro S, et al. Assessing appendicular skeletal muscle mass with bioelectrical impedance analysis in free-living Caucasian older adults. Clin Nutr. (2015) 34:667-73. doi: 10.1016/j.clnu.2014.07.010

37. Dekker MJE, Konings C, Canaud B, van der Sande FM, Stuard S, Raimann JG, et al. Interactions between malnutrition, inflammation, and fluid overload and their associations with survival in prevalent hemodialysis patients. J Ren Nutr. (2018) 28:435-44. doi: 10.1053/j.jrn.2018.06.005

38. Wu B, Yan C, Zhang S, Ge Y, Xu X, Wang Y, et al. Comparative performance of body composition parameters in prediction of death in hospitalized patients on maintenance hemodialysis: a cohort study. Sci Rep. (2020) 10:10199. doi: 10.1038/s41598-020-67019-0

39. Hwang SD, Lee JH, Lee SW, Kim JK, Kim MJ, Song JH. Risk of overhydration and low lean tissue index as measured using a body composition monitor in patients on hemodialysis: a systemic review and meta-analysis. Ren Fail. (2018) 40:51-59. doi: 10.1080/0886022X.2017.1419963

40. Zhou Y, Hoglund P, Clyne N. Comparison of DEXA and bioimpedance for body composition measurements in nondialysis patients with CKD. J Ren Nutr. (2019) 29:33-8. doi: 10.1053/j.jrn.2018.05.003

41. Lim PS, Chen CH, Zhu F, Kotanko P, Jeng Y, Hu CY, et al. Validating body fat assessment by bioelectric impedance spectroscopy in Taiwanese hemodialysis patients. J Ren Nutr. (2017) 27:37-44. doi: 10.1053/j.jrn.2016.08.003

42. Ikizler TA, Burrowes JD, Byham-Gray LD, Campbell KL, Carrero JJ, Chan W, et al. KDOQI clinical practice guideline for nutrition in CKD: 2020 update. Am J Kidney Dis. (2020) 76(3 Suppl. 1):S1-107. doi: 10.1053/j.ajkd.2020.05.006

43. Keane DF, Baxter P, Lindley E, Moissl U, Pavitt S, Rhodes L, et al. The body composition monitor: a flexible tool for routine fluid management across the haemodialysis population. Biomed Phys Eng Exp. (2017) 3:035017. doi: 10.1088/2057-1976/aa6f45

44. Dekker M, Konings C, Canaud B, Carioni P, Guinsburg A, Madero M, et al. Pre-dialysis fluid status, pre-dialysis systolic blood pressure and outcome in prevalent haemodialysis patients: results of an international cohort study on behalf of the MONDO initiative. Nephrol Dial Transplant. (2018) 33:2027-34. doi: 10.1093/ndt/gfy095

45. Sheean P, Gonzalez MC, Prado CM, McKeever L, Hall AM, Braunschweig CA. American society for parenteral and enteral nutrition clinical guidelines: the validity of body composition assessment in clinical populations. J Parenter Enteral Nutr. (2020) 44:12-43. doi: 10.1002/jpen.1669

46. Silva AM, Matias CN, Nunes CL, Santos DA, Marini E, Lukaski HC, et al. Lack of agreement of in vivo raw bioimpedance measurements obtained from two single and multi-frequency bioelectrical impedance devices. Eur J Clin Nutr. (2019) 73:1077-83. doi: 10.1038/s41430-018-0355-Z

47. Patel SS, Molnar MZ, Tayek JA, Ix JH, Noori N, Benner D, et al. Serum creatinine as a marker of muscle mass in chronic kidney disease: results of a cross-sectional study and review of literature. J Cachexia Sarcopenia Muscle. (2013) 4:19-29. doi: 10.1007/s13539-012-0079-1

48. Park J, Mehrotra R, Rhee CM, Molnar MZ, Lukowsky LR, Patel SS, et al. Serum creatinine level, a surrogate of muscle mass, predicts mortality in peritoneal dialysis patients. Nephrol Dial Transplant. (2013) 28:2146-55. doi: $10.1093 / \mathrm{ndt} / \mathrm{gft} 213$

49. Canaud B, Granger Vallee A, Molinari N, Chenine L, Leray-Moragues H, Rodriguez $\mathrm{A}$, et al. Creatinine index as a surrogate of lean body mass derived from urea $\mathrm{Kt} / \mathrm{V}$, pre-dialysis serum levels and anthropometric 
characteristics of haemodialysis patients. PLoS ONE. (2014) 9:e93286 doi: 10.1371/journal.pone.0093286

50. Terrier N, Jaussent I, Dupuy AM, Morena M, Delcourt C, Chalabi L, et al. Creatinine index and transthyretin as additive predictors of mortality in haemodialysis patients. Nephrol Dial Transplant. (2008) 23:345-53. doi: $10.1093 /$ ndt/gfm573

51. Canaud B, Ye X, Usvyat L, Kooman J, van der Sande F, Raimann J, et al. Clinical and predictive value of simplified creatinine index used as muscle mass surrogate in end-stage kidney disease haemodialysis patients-results from the international MONitoring Dialysis Outcome initiative. Nephrol Dial Transplant. (2020) 35:2161-71. doi: 10.1093/ndt/gfaa098

52. Ye X, Dekker MJE, Maddux FW, Kotanko P, Konings C, Raimann JG, et al. Dynamics of nutritional competence in the last year before death in a large cohort of US hemodialysis patients. J Ren Nutr. (2017) 27:412-20. doi: 10.1053/j.jrn.2017.06.006

53. Thijssen S, Wong MM, Usvyat LA, Xiao Q, Kotanko P, Maddux FW. Nutritional competence and resilience among hemodialysis patients in the setting of dialysis initiation and hospitalization. Clin J Am Soc Nephrol. (2015) 10:1593-601. doi: 10.2215/CJN.08430814

54. Ye X, Kooman JP, van der Sande FM, Raimann JG, Usvyat LA, Wang Y, et al. Relationship between serum phosphate levels and survival in chronic hemodialysis patients: interactions with age, malnutrition and inflammation. Clin Kidney J. (2021) 14:348-57. doi: 10.1093/ckj/sfz143

55. Beaudart C, McCloskey E, Bruyere O, Cesari M, Rolland Y, Rizzoli R, et al. Sarcopenia in daily practice: assessment and management. BMC Geriatr. (2016) 16:170. doi: 10.1186/s12877-016-0349-4

56. Sabatino A, Regolisti G, Bozzoli L, Fani F, Antoniotti R, Maggiore U, et al. Reliability of bedside ultrasound for measurement of quadriceps muscle thickness in critically ill patients with acute kidney injury. Clin Nutr. (2017) 36:1710-15. doi: 10.1016/j.clnu.2016.09.029

57. Sabatino A, Regolisti G, Delsante M, Di Motta T, Cantarelli C, Pioli S, et al. Noninvasive evaluation of muscle mass by ultrasonography of quadriceps femoris muscle in end-stage renal disease patients on hemodialysis. Clin Nutr. (2019) 38:1232-9. doi: 10.1016/j.clnu.2018.05.004

58. Connolly B, MacBean V, Crowley C, Lunt A, Moxham J, Rafferty GF, et al. Ultrasound for the assessment of peripheral skeletal muscle architecture in critical illness: a systematic review. Crit Care Med. (2015) 43:897-905. doi: 10.1097/CCM.0000000000000821

59. Ticinesi A, Meschi T, Narici MV, Lauretani F, Maggio M. Muscle ultrasound and sarcopenia in older individuals: a clinical perspective. J Am Med Dir Assoc. (2017) 18:290-300. doi: 10.1016/j.jamda.2016.11.013

60. Sabatino A, di Mario F, Ciuni A, Palumbo A, Peyronel F, Maggiore U, et al. Validation by CT scan of quadriceps muscle ultrasound in acute kidney injury. J Nephrol. (2020) 33:109-17. doi: 10.1007/s40620-019-00659-2

61. Souza VA, Oliveira D, Cupolilo EN, Miranda CS, Colugnati FAB, Mansur $\mathrm{HN}$, et al. Rectus femoris muscle mass evaluation by ultrasound: facilitating sarcopenia diagnosis in pre-dialysis chronic kidney disease stages. Clinics. (2018) 73:e392. doi: 10.6061/clinics/2018/e392

62. Sahathevan S, Khor B, Singh B, Sabatino A, Fiaccadori E, Daud Z, et al. Association of ultrasound-derived metrics of the quadriceps muscle with protein energy wasting in hemodialysis patients: a multicenter cross-sectional study. Nutrients. (2020) 12:3597. doi: 10.3390/nu12113597

63. Sabatino A, Maggiore U, Regolisti G, Rossi GM, Di Mario F, Gentile M, et al. Ultrasound for non-invasive assessment and monitoring of quadriceps muscle thickness in critically ill patients with acute kidney injury. Front Nutr. (2021) 8:622823. doi: $10.3389 /$ fnut.2021.622823

64. Gould D, Watson E, Wilkinson T, Wormleighton J, Xenophontos S, Viana J, et al. Ultrasound assessment of muscle mass in response to exercise training in chronic kidney disease: a comparison with MRI. J Cachexia Sarcopenia Muscle. (2019) 10. doi: 10.1002/jcsm. 12429

65. Isoyama N, Qureshi AR, Avesani CM, Lindholm B, Barany P, Heimburger $\mathrm{O}$, et al. Comparative associations of muscle mass and muscle strength with mortality in dialysis patients. Clin J Am Soc Nephrol. (2014) 9:1720-8. doi: 10.2215/CJN.10261013

66. Broers NJH, Fung TY, Kooman JP, Christiaans MHL. Living-donor transplantation leads to a major improvement in physical functioning: an observational study on the impact on potential donors and their recipients. BMC Nephrol. (2019) 20:109. doi: 10.1186/s12882-019-1299-9

67. Broers NJ, Usvyat LA, Kooman JP, van der Sande FM, Lacson E Jr, Kotanko P, et al. Quality of life in dialysis patients: a retrospective cohort study. Nephron. (2015) 130:105-12. doi: 10.1159/000430814

68. Huang M, Lv A, Wang J, Xu N, Ma G, Zhai Z, et al. Exercise training and outcomes in hemodialysis patients: systematic review and meta-analysis. Am J Nephrol. (2019) 50:240-54. doi: 10.1159/000502447

69. Dirks ML, Hansen D, Van Assche A, Dendale P, Van Loon JL. Neuromuscular electrical stimulation prevents muscle wasting in critically ill comatose patients. Clin Sci. (2015) 128:357-65. doi: 10.1042/CS201 40447

70. John B, Tan BK, Dainty S, Spanel P, Smith D, Davies SJ. Plasma volume, albumin, and fluid status in peritoneal dialysis patients. Clin J Am Soc Nephrol. (2010) 5:1463-70. doi: 10.2215/CJN.09411209

Conflict of Interest: The authors declare that the research was conducted in the absence of any commercial or financial relationships that could be construed as a potential conflict of interest.

Publisher's Note: All claims expressed in this article are solely those of the authors and do not necessarily represent those of their affiliated organizations, or those of the publisher, the editors and the reviewers. Any product that may be evaluated in this article, or claim that may be made by its manufacturer, is not guaranteed or endorsed by the publisher.

Copyright (๔ 2021 Sabatino, Broers, van der Sande, Hemmelder, Fiaccadori and Kooman. This is an open-access article distributed under the terms of the Creative Commons Attribution License (CC BY). The use, distribution or reproduction in other forums is permitted, provided the original author(s) and the copyright owner(s) are credited and that the original publication in this journal is cited, in accordance with accepted academic practice. No use, distribution or reproduction is permitted which does not comply with these terms. 